\title{
Assessing commercially available personal health records for home health
}

\section{Recommendations for design}

Laura Kneale ${ }^{1}$; Yong Choi'; George Demiris ${ }^{1,2}$

${ }^{1}$ University of Washington Biomedical and Health Informatics;

${ }^{2}$ University of Washington School of Nursing

\section{Keywords}

Personal health record, personal health records, home health nursing, qualitative research

\section{Summary}

Background: Home health nurses and clients experience unmet information needs when transitioning from hospital to home health. Personal health records (PHRs) support consumer-centered information management activities. Previous work has assessed PHRs associated with healthcare providers, but these systems leave home health nurses unable to access necessary information. Objectives: To evaluate the ability of publically available PHRs to accept, manage, and share information from a home health case study.

Methods: Two researchers accessed the publically available PHRs on myPHR.com, and attempted to enter, manage, and share the case study data. We qualitatively described the PHR features, and identified gaps between the case study information and PHR functionality.

Results: Eighteen PHRs were identified in our initial search. Seven systems met our inclusion criteria, and are included in this review. The PHRs were able to accept basic medical information. Gaps occurred when entering, managing, and/or sharing data from the acute care and home health episodes. The PHRs that were reviewed were unable to effectively manage the case study information. Therefore, increasing consumer health literacy through these systems may be difficult. The PHRs that we reviewed were also unable to electronically share their data.

Conclusions: The gap between the existing functionality and the information needs from the case study may make these PHRs difficult to use for home health environments. Additional work is needed to increase the functionality of the PHR systems to better fit the data needs of home health clients.

\section{Correspondence to:}

Laura Kneale

Biomedical and Health Informatics

Department of Biomedical Informatics and Medical

Education

University of Washington

Box 358047

Seattle, WA 98195

Email: Ikneale@uw.edu
Appl Clin Inform 2016; 7: 355-367

received: November 20, 2015

accepted: February 28, 2016

published: May 18, 2016

http://dx.doi.org/10.4338/ACl-2015-11-RA-0156

Citation: Kneale L, Choi Y, Demiris G. Assessing commercially available personal health records : recommendations for design. Appl Clin Inform 2016; 7: 355-367

http://dx.doi.org/10.4338/ACl-2015-11-RA-0156 


\section{Background}

A recent survey conducted by the Commonwealth Fund reported that $35 \%$ of older adult Americans faced serious challenges with care coordination. Among the issues reported, $16 \%$ received conflicting information from their doctors, 19\% thought that their specialists and/or regular doctors lacked the clinical data required to be effective, $28 \%$ experienced gaps in hospital discharge planning, and $11 \%$ positively responded to the statement "doctors seemed uninformed about hospital care after discharge in the past 2 years" [1]. Unfortunately, these challenges are seen across many care settings. For example, home health nurses, older adults, and informal caregivers have reported experiencing unmet information needs during the hospital to home health care transition [2-4]. This lack of information during the hospital to home health handoff can reduce home health provider's ability to provide evidence-based disease management in the home [4].

Personal health records (PHRs) are tools that have been developed to support consumer-driven health information management, and facilitate information exchange. The Markle Foundation describes a PHR as "an electronic application through which individuals can access, manage, and share their health information, and that of others for whom they are authorized, in a private, secure, and confidential environment" [5]. In the United States, there are two models of PHRs: tethered and standalone. Tethered personal health records are only available to consumers who are part of a specific healthcare system and/or insurance network. Typically, these systems are free to the healthcare consumers within a system, and the costs associated with development and maintenance fall on the healthcare system and/or insurance agency. Standalone personal health records are open to any consumer who creates an account. These PHRs may be developed by a commercial or non-profit organization, and the costs associated with development and maintenance are either collected from the end user through a payment system or generated by other revenue sources [6,7].

In a recent study of the U.S. Veteran's Administration's My HealtheVet PHR, older adult veterans reported that PHR use led to a greater understanding of their own health information. In addition, many participants stated that their care team was better prepared to provide care due to the increased information sharing [8]. Unfortunately tethered PHRs, like the My HealtheVet system, exclude non-network providers from the system. This exclusion of non-network providers may be a significant challenge for home health care environments. In 2009, 85\% of Medicare-certified home health agencies were not affiliated with hospitals, rehabilitation centers, and/or skilled nursing facilities [9]. These freestanding home health agencies are excluded from the information available in tethered PHR systems. This exclusion may continue to promote fragmented communication practices, and continue the challenge with unmet information needs.

\section{Objectives}

Our study aims to analyze publically available personal health records for their suitability to accept, manage, and share data generated from a published home health case study. Specifically, our findings answer four questions: 1) is there a location in the PHR to enter and store the data from the case study, 2) how can the data be updated to reflect changes in the clinical data over time, 3) are consumers able to make connections between data to better understand current and/or past medical information, and 4) what mechanisms allow consumers to import and export data in electronic formats? From our findings, we recommend design changes that would enhance existing PHRs suitability to accept, manage, and share data from home health encounters.

\section{Methods}

\subsection{PHR search strategy}

We used a personal health record consumer advocate website, MyPHR.com, to gather a list of available personal health records. MyPHR.com is managed by the American Health Information Management Association, and is advertised as a consumer resource for locating and choosing personal 
health records [6]. The MyPHR.com database was searched under the "Choose a PHR" web page using the standard filters: "Web-based" for format, and "Free" for cost in June 2015 [10]. This database resource was chosen as it targets healthcare consumers, and it has been used in previous personal health record systematic reviews [11]. We chose to filter for no-cost personal health records because previous research suggested that cost is a barrier for adopting a PHR system [12].

\subsection{Home health case study}

Our case study was created from a published medical case study describing a 58-year-old man referred to home health after a new diagnosis of congestive heart failure (CHF) in an acute care setting [13]. Information from the published case study was abstracted into four categories: demographic information, medical history, acute care data, and home health encounters. The home health data included an initial home health assessment and plan of care, daily weight recordings by the patient, two management changes from subsequent home health visits, and a final assessment before the patient was readmitted to an acute care setting for uncontrolled CHF. Prior to the acute care admission, this patient had four co-morbidities (hypertension, type II diabetes, ischemic heart disease, and atrial fibrillation), and regularly took five medications [14].

This medical history is consistent with the overall health profile of older adult Americans. A recent report from the Administration on Aging states that most American older adults have more than one co-morbidity, and heart disease, hypertension, and diabetes rank among the most common conditions [14]. In addition, this patient was readmitted to the hospital during the home health encounter. As other research has reported, CHF 30-day readmissions account for the most common Medicare 30-day readmission diagnosis, and 24.5\% of Medicare recipients discharged from an acute care stay with a primary diagnosis of CHF are readmitted within 30 days [15].

\subsection{PHR assessment}

The primary author (LK) accessed the website address provided from MyPHR.com for each PHR in the search results. Internet searches were completed if the website from the database was incorrect. We used the PHR name from the MyPHR.com database as the search term. When a website address was identified, the authors reviewed the site for inclusion. PHRs were excluded if the system was not easily available to the public, and/or if the PHR did not meet the Markle Foundation's definition of a personal health record. Tethered PHRs were excluded because these systems were not considered publically available. There were four reasons for exclusion: failure to locate an active web address, PHR services had been discontinued, restricted consumer access, and the PHR functionality did not provide the user ability to access, manage, and share their health information over the Internet.

Two authors (LK and YC) independently reviewed each of the included PHR systems in August and September 2015. Results from the assessments were independently recorded using a Microsoft Excel spreadsheet. Each researcher also recorded qualitative observations about the ease of use, difficulties encountered performing the tasks, and general observations about the PHR functionality. After the independent review, the authors met to analyze the data, review differences in abstraction observations, and qualitatively describe the functionality of the reviewed systems.

\section{Results}

Eighteen PHRs were identified from the MyPHR.com database search. Eleven PHRs were excluded from this analysis. Of these initial PHRs, the authors were unable to locate the website of one vendor using Internet searches. Two PHRs have discontinued their services [16, 17]. Four PHRs had a tethered model [18-21], and three did not meet our definition of a personal health record [22-24]. Specifically, one system was a United Kingdom-based non-profit site that curated patient health education [22], the second system was a company that sold medical emergency wearable devices with a companion website [23], and the third system was a diabetes social and education network [24]. Finally, one PHR was excluded because it required participation in a sales call before creating an account [25]. 


\subsection{Is there a location to enter and store data abstracted from the case study?}

For this review, we attempted to enter six categories of data from the clinical case study into each PHR. An overview of the results from our analysis is shown in Table 1.

\subsubsection{Demographics}

All seven (100\%) of the PHRs reviewed were able to accept the basic information from the demographics in structured or free text formats [26-32]. Basic demographic information included date of birth, race and ethnicity, medical insurance information, current tobacco and alcohol use, and social history. One challenge occurred when attempting to enter information related to the patient's living situation and occupational therapy data. Our case study described the patient's home environment, including the number of steps needed to access his apartment [13]. Three of the seven (43\%) PHRs were able to accept this information in free text formats $[26,27,31]$; however, none of the PHRs reviewed offered pre-defined labels to accept this information.

\subsubsection{Medical History}

All systems were able to accept the basic medical history in a text format including medications, previous medical conditions, and basic demographics. Medical history information included current diagnoses of hypertension, type II diabetes, and ischemic heart disease, a previous coronary artery bypass graft with no complications, five daily medications, and three clinical providers [13].

Despite the ease of finding the appropriate location for the data elements, the authors experienced challenges with entering the medical history information. Four PHRs (57\%) used predetermined lists to enter medications, current conditions, and previous procedures [28, 29, 31, 32]; however, the available medical terms varied in the data entry lists. For example, the term "ischemic heart disease" was not available in two of the four PHRs that used drop-down lists for medical history data entry $[31,32]$. Therefore, the authors had to use Internet searches to determine the most appropriate synonym that was available in the system. When exact terms could not be located, the authors resorted to using free text fields to clarify or add additional information to the disease.

\subsubsection{Acute Care Data}

The acute care data included emergency room clinical assessments, laboratory values, an echocardiogram, and a chest $\mathrm{x}$-ray. In addition, the patient's care plan was dynamic and required multiple medication adjustments throughout the seven day hospitalization. The patient was discharged with an outpatient follow up appointment, and referred to a home health service [13].

Basic acute care data such as medications, patient vitals, laboratory values, and new diagnoses could be entered into all the PHRs. For example, medication changes were added to the global medication list, and vital signs were included with the pre-hospitalization values. Three PHRs (43\%) accepted information about future medical appointments [28-30], and one of these systems provided the functionality for consumers to set up reminders for these appointments [30]. Additionally, one system (14\%) allowed the user to directly communicate with their provider and to request future appointments through the PHR interface [32]. None of the systems that we reviewed had dedicated structured or free-text fields for clinician notes/assessments, test results from the echocardiogram, and chief complaints from the emergency room; however, six of the seven PHRs (86\%) allowed users to upload portable document format (PDF) summaries of clinical information [26-31].

\subsubsection{Home Health Encounters}

The home health data included an initial assessment and plan of care, daily weight recordings by the patient, two medication dose changes, and a final clinical assessment [13]. Similar to the acute care data, the basic medical information from the home health episode could be entered into structured fields into five of the personal health records [26-29, 31]. One PHR (14\%) did not accept daily weight measurements [32]. In addition, the home health nurse offered her clinical assessment of the clinical data, such as categorizing the patient as hypotensive and bradycardic after taking vital readings [13]. Six of the seven PHRs (86\%) were able to accept the judgments as free text data elements associated with the numerical vital values $[26-30,32]$.

(c) Schattauer 2016 L. Kneale, Y. Choi, G. Demiris: Assessing commercially available personal health records 
The clinical home health data, including nursing assessments, were unable to be entered into structured data fields. Additionally, none of the PHRs reviewed offered dedicated areas to enter and store plan of care or patient instruction documents. Although there were no dedicated areas, two PHRs (29\%) offered a journal or a health diary that could be used for this purpose [30, 31], and another system (14\%) offered a place to store generic "short notes" [27].

\subsection{How can data be updated to reflect changes to clinical information over time?}

The authors focused on the data management of three data elements: the medication list, daily weight readings, and laboratory values. Five PHRs (71\%) were able to accept daily weights values, medications, and laboratory values [26-29, 31]. One system (14\%) was only able to accept daily weight measurements and medication information [30], and another system (14\%) did not accept weight values [32]. An overview of our results is displayed in $>$ Table 2.

To manage the medication information, the authors attempted to update dosages of a medication multiple times to reflect the frequent changes to the medication list in the case study. Two PHRs (29\%) allowed the user to change the dose of an existing medication entry, while maintaining a record of the previous dosage value. However, in these systems the record of the previous dose change was displayed in the separate systems log, which detailed every action in the PHR. Therefore, if users wanted to identify historical medication dose changes, the user had to access two separate locations in the PHR $[29,30]$. Four systems (57\%) required the user to create an entirely new medication entry to update the dose information, while maintaining a history of the previous value [26-31]. One system (14\%) was only able to keep an active medications list, and did not store information related to discontinued or deleted medications [32].

\subsection{Are consumers able to make connections between data to better understand current and/or past medical history?}

Six of the systems (86\%) provided a way for consumers to view their longitudinal weight data. Three PHRs offered consumers the choice to view their measurements in a table or a line graph format [26, 29, 30], and three PHRs only displayed table views of this information [27, 28, 31]. In addition, five systems (71\%) allowed users to track laboratory data, and associate the data with a specific date and time. Two of these systems only allowed specific values to be captured and tracked in the system [26, 27], and three systems allowed the user to specify any numerical value to track [28, 29, 31].

\subsection{What mechanisms allow consumers to import and export data in electronic formats?}

Finally, we analyzed the available mechanisms to import and export the PHR data in electronic formats. Our results from this analysis are shown in $>$ Table 3. Five of the seven PHRs (71\%) allowed access to external parties [26, 28-31]. Four of these PHRs (80\%) allowed the consumer to specify what information is accessible to the external parties either through setting role based privacy rules, or by hiding data fields from external parties [26, 28-30]. In addition, four systems (57\%) offered secure messaging through the system [26-29], and one system (14\%) provided the functionality for the user to attach specific PHR data elements directly into a secure message [29].

All seven PHRs (100\%) provided a way to export the health record data. Four PHRs (57\%) only offered a printer-friendly version and/or portable document format (PDF) of the data [27, 28, 30, 32]. The remaining three PHRs (43\%) offered a variety of standard documents, in addition to providing a PDF version of the content. Two systems provided continuity of care document (CCD), and continuity of care record (CCR) export formats [26, 29]. The third system offered CCD and consolidated clinical document architecture (C-CDA) export formats [31]. The PHRs did not specify what release number(s) were supported by the export features. In addition, one system (14\%) also pro- 
vided two other export formats: Plain Text/ASCII format ("Blue Button"), and a "PHR format", an Extensible Markup Language (XML) file [26]. Despite the overlap in electronic standards, when we attempted to move the clinical data from one PHR system to another using the data sharing standards, we found that none of the PHRs that we reviewed were able to accept data from another PHR except through PDF documents.

\section{Discussion}

Consumers referred to home health following an acute care stay have unique health data. Not only do these consumers have clinical data from their acute care and home health encounters, but their medical data also includes personalized care plans and patient reported outcomes. Overall the PHRs analyzed in this study faced challenges in accepting, managing, and electronically sharing information from our home health case study. Our study highlights additional functionality that would enhance the suitability of existing PHRs to accept, manage, and share home health data.

Although our study focused on standalone PHRs, our findings were consistent with other recently published studies analyzing tethered PHR functionality. For example, researchers interviewed 16 organizations that provided tethered PHRs for their patients. This study found that these PHRs were an underutilized tool for patients with chronic diseases, and called for increased focus on patient-centered tools to support engagement and shared decision-making [33]. Our findings suggest that these gaps are also found in standalone PHRs, and more work is needed to maximize the utility of these systems.

\subsection{Is there a location to enter and store data abstracted from the case study?}

The ability to enter and store health data is a core function of a personal health record [5]. All seven systems were able to accept basic medical information including medications, medical history, and most accepted laboratory values into structured data fields. A gap in functionality occurred when entering data related to the acute care and home health encounters. Test results, provider notes, and clinical summaries did not have dedicated data fields, and could only be entered into the system by uploading a PDF document with this information. In addition, none of the PHRs had dedicated areas to store information related to discharge instructions or patient care plans.

Patient reported outcomes (PROs) and consumer health information are also important components of successful PHR systems [5, 7], and every home health encounter is centered around a care plan that is uniquely designed to meet the client's clinical needs [3]. Therefore, it was surprising that the seven PHRs were unable to store and manage an individual care plan. This gap in functionality would require PHR users to store patient instructions in a different location then where they monitor their care plan.

Finally, the PHRs that we reviewed did not facilitate the documentation of consumer care goals, and/or treatment preferences. As other research suggests, PHRs could be a key tool in facilitating patient-centered care for people with chronic diseases [33]. One way to accomplish this would be to use PHRs as a place for consumers to explore and document data related to shared decision-making processes, such as patient treatment preferences. Unfortunately, the current functionality of available PHRs does not support this type of data collection.

In order for PHRs to be better suited to accept the medical history of older adult home health service recipients, PHRs should develop structured data fields for information related to clinical encounters including test results, provider assessments, and individual care plans. In addition, adding database fields for consumers and caregivers to document care goals, treatment preferences, and values could help PHRs promote more patient-centered care. 


\subsection{How can data be updated to reflect changes to clinical information over time?}

As exemplified in our home health case study, changes to medical routines are common during the hospital to home health care transition [13]. The PHRs that we reviewed were not able to easily accept changes to existing clinical information, such as a dose change to a medication record. In order to make these changes and keep a record of the previous dose, the user had to discontinue the existing medication entry and create new medication record.

To increase usefulness, PHRs need to adopt more usable ways to update medical information with changing care plans. Maintaining a historical record of medications is valuable to understand how medication changes affect patient conditions; however, creating several new medication entries each time a dose or medication timing changes may be overwhelming in the long term. Re-entering meds each time may also increase the opportunity for data entry errors. To be a true longitudinal care record, PHRs should develop ways to assist consumers with making care plan modifications, while also allowing them to understand the global changes to their medications over time.

\subsection{Are consumers able to make connections between data to better understand current and/or past medical history?}

Our case study described a patient's worsening condition over the course of several home health visits. The home health nurse required several visits, clinical assessments, and patient inquiries to determine that medication changes were the probable cause of the decline in the patient condition [13]. Unfortunately, none of the systems that we reviewed allowed consumers to relate medications information to patient reported outcomes. If available, this additional feature may have helped the consumer realize that their medication changes may be the source of their increased shortness of breath, and alert their providers of this observation. Although both the medication changes and shortness of breath data elements were available in the PHR system, significant manual effort would be required to relate these data points with each other. Similarly, gaining greater understanding of the content of medical data, such as vitals or laboratory results, would be difficult for the end user. Even though each vital and lab value was linked to a date/time stamp, a record of the setting where this data point was acquired (e.g., home health, clinic or acute care) was not available, potentially limiting the ability to fully interpret these clinical data elements.

A goal of PHRs should be to help patients "create a sense of the patient journey over time" [33]. By having separate areas to store categories of structured medical data, PHRs are reducing the effectiveness of having this data in electronic, structured fields. In order to increase the impact of PHR data on the consumer, we suggest that PHRs should focus on additional functionality to help consumers relate aspects of their medical history in order to gain a better overall sense of their medical data.

\subsection{What mechanisms allow consumers to import and export data in electronic formats?}

One of the ways to reduce the burden on the consumer or caregiver in entering clinical data is to develop mechanisms to electronically import and export clinical data from one system to another. Four of the seven systems (57\%) would only accept outside data as a PDF or through manual data entry. Three systems presented the ability to import and export data using health information data standards [26, 29, 31]; however, when we tested this functionality, we found that none of the systems were able to successfully accept data from another PHR.

As the Meaningful Use (MU) stage 2 law advises, electronic import and export mechanisms is critical for successful communication in our siloed healthcare system. If the PHRs were able to electronically move data into and out of the system, it would promote better information sharing across care teams. Recent work has suggested that the MU standards for interoperability of health records may be hindering the goal of universal, consumer-friendly interoperability. This study found that 
the rush to implement PHRs within healthcare systems is leaving patients forced to manage multiple PHRs without the opportunity to consolidate clinical data [34]. Our findings support this work by showing that PHRs, using their current standards, do not accept electronic data from each other. More work is needed to ensure that data standards are implemented to facilitate easy electronic data sharing with consumers' electronic health records, and other tethered and standalone PHRs.

\subsection{Usability}

Although we did not formally assess usability, we found these systems had non-intuitive interfaces. Most of the inconsistencies during the data collection process happened because one reviewer did not find the functionality due to the poor user interfaces. For example, one PHR had the health diary feature under the "print" drop down menu [31]. Therefore, if end users do not go through each of the drop down menus, he or she may miss the health diary feature. Additionally, robust help features and how-to-use tutorials in the PHRs, or on the public websites, were rare. In order to improve the ability of end-users to learn to use the systems, more work is needed to ensure adequate help is available through the PHR websites. Older adults have been adopting technology at increasing rates, however, the older users still lag behind younger users [35]. PHR evaluations have found usability issues to be a consistent barrier to usage among older adults $[8,12,36]$. Our findings indicate that future studies should include a formal usability evaluation on PHRs for older adult home health consumers.

\subsection{Limitations}

Our study focused on personal health records that were publically available to consumers and independent home health providers. Due to the restrictions on public access to tethered and fee-based systems, we were only able to review no-cost, standalone PHRs. Although these restrictions were chosen to maximize the feasibility of using the PHR systems in home health environments, it is possible that tethered and/or fee-based PHRs are better suited for the data in our case study. Therefore, we advocate future studies include fee-based and tethered PHRs where possible to determine our findings are consistent across all PHR sub-groups.

In addition, we used expert review to analyze the PHRs for this study. This methodology was used to ensure consistency of analysis, and to fit within our budget restraints. This choice was necessary for our study, but leaves out the important consumer perspective. To better understand how the limitations that we found in this study affect home health consumers and their home health providers, future studies should include these end-user groups in analyses of PHRs in home health environments.

\section{Conclusion}

Today's older adults in home health care settings have complex care needs, interact with multiple providers from different healthcare networks, and experience unmet information needs. Participants in the hospital to home health care transitions are particularly vulnerable to gaps in information due to the fragmented provider-centered communication patterns [2-4]. Personal health records have been shown to facilitate information sharing and support increased consumer engagement in other care settings $[8,12,37]$. Our findings suggest that there are functionality gaps with the existing publically available PHRs. In this study, we highlight additional functionality that would make the current PHR tools more valuable for consumers that receive home health services following an acute care stay. Home health is quickly moving towards health information technology solutions to increase care coordination, promote evidence based care, and capture important clinical information [38]. Our findings suggest that with changes to existing systems, PHRs could help reduce the health information needs of home health clients, caregivers, and nurses. 


\section{Clinical Relevance Statement}

Home care nurses, clients, and caregivers experience unmet information needs during transitions from the hospital to home care. Personal health records (PHRs) are one tool that may help facilitate information sharing, and this study investigated how existing publically available PHRs are able to accept, manage, and share clinical information in a home health case study. The gaps that we found between current PHR functionality and information generated in home health encounters may reduce the effectiveness of these tools for home health clients and providers

\section{Conflict of Interest}

The authors have no conflicts of interest to report.

\section{Human Subject Protections}

Human and/or animal subjects were not included in this study.

\section{Acknowledgements}

The authors would like to thank the Home Health and Hospice Information Technology (H3IT) Conference for the opportunity to present this work. This study was supported in part by the National Library of Medicine Biomedical and Health Informatics Training Grant at the University of Washington Grant Nr. T15LM007442. 
Table 1 Predefined Database Fields in the PHRs

\begin{tabular}{|c|c|c|c|c|c|c|}
\hline \multirow[t]{2}{*}{ PHR Name } & \multicolumn{6}{|c|}{ Predefined Database Fields } \\
\hline & $\begin{array}{l}\text { Demo- } \\
\text { graphics }\end{array}$ & $\begin{array}{l}\text { Occupa- } \\
\text { tional Ther- } \\
\text { apy Data }\end{array}$ & $\begin{array}{l}\text { Previous } \\
\text { Medical } \\
\text { History }\end{array}$ & $\begin{array}{l}\text { Acute } \\
\text { Care Data }\end{array}$ & $\begin{array}{l}\text { Home } \\
\text { Health Data }\end{array}$ & $\begin{array}{l}\text { Patient } \\
\text { Reported } \\
\text { Outcomes }\end{array}$ \\
\hline $\begin{array}{l}\text { No More Clip- } \\
\text { board }\end{array}$ & $x$ & $x$ & $x$ & & & $x$ \\
\hline Healthspek & $x$ & $x$ & $x$ & & & $x$ \\
\hline Health Companion & $x$ & & $x$ & & & \\
\hline HealthVault & $x$ & & $x$ & & & $x$ \\
\hline Remember It Now & $x$ & & $x$ & & & $x$ \\
\hline MyMed Wall & $X$ & $\mathrm{X}$ & $\mathrm{X}$ & & & $x$ \\
\hline $\begin{array}{l}\text { My Doclopedia } \\
\text { PHR }\end{array}$ & $x$ & & $x$ & & & \\
\hline
\end{tabular}

Table 2 PHR Functions to Manage Clinical Data

\begin{tabular}{|l|l|l|l|}
\hline PHR Name & $\begin{array}{l}\text { Change Medication } \\
\text { Dose }\end{array}$ & $\begin{array}{l}\text { View Longitudinal } \\
\text { Daily Weight Values }\end{array}$ & Track Laboratory Values \\
\hline No More Clipboard & $\begin{array}{l}\text { Must discontinue medi- } \\
\text { cation to maintain record of } \\
\text { previous dose }\end{array}$ & View in table or line graph & $\begin{array}{l}\text { Track only predefined lab } \\
\text { values }\end{array}$ \\
\hline Healthspek & $\begin{array}{l}\text { Must discontinue medi- } \\
\text { cation to maintain record of } \\
\text { previous dose }\end{array}$ & View in table only & $\begin{array}{l}\text { Track only predefined lab } \\
\text { values }\end{array}$ \\
\hline Health Companion & $\begin{array}{l}\text { Must discontinue medi- } \\
\text { cation to maintain record of } \\
\text { previous dose }\end{array}$ & View in table only & Track any lab value \\
\hline HealthVault & $\begin{array}{l}\text { Maintained record of pre- } \\
\text { vious dose in systems log }\end{array}$ & View in table or line graph & Track any lab value \\
\hline Remember It Now & $\begin{array}{l}\text { Maintained record of pre- } \\
\text { vious dose in systems log }\end{array}$ & View in table or line graph & Does not accept lab values \\
\hline MyMed Wall & $\begin{array}{l}\text { Must discontinue medi- } \\
\text { cation to maintain record of } \\
\text { previous dose }\end{array}$ & View in table only & Track any lab value \\
\hline My Doclopedia PHR & $\begin{array}{l}\text { Does not maintain record of } \\
\text { previous medications/doses }\end{array}$ & $\begin{array}{l}\text { Does not accept daily } \\
\text { weight values }\end{array}$ & Does not accept lab values \\
\hline
\end{tabular}


Table 3 Electronic Mechanisms to Share PHR Data

\begin{tabular}{|c|c|c|c|c|}
\hline PHR Name & $\begin{array}{l}\text { Grant Access to Ex- } \\
\text { ternal Parties }\end{array}$ & $\begin{array}{l}\text { Secure Messag- } \\
\text { ing through PHR }\end{array}$ & Import Standards & $\begin{array}{l}\text { Export Stan- } \\
\text { dards }\end{array}$ \\
\hline No More Clipboard & Role-based access & $x$ & $\begin{array}{l}\text { CCR, CCD, ASCII, PHR } \\
\text { format, PDF }\end{array}$ & $\begin{array}{l}\text { PDF, CCR, CCD, } \\
\text { ASCII, PHR } \\
\text { format }\end{array}$ \\
\hline Healthspek & & $X$ & & PDF \\
\hline Health Companion & Role-based access & $X$ & PDF & PDF \\
\hline HealthVault & Role-based access & $X$ & $C C D, C C R, P D F$ & PDF, CCD, CCR \\
\hline Remember It Now & Role-based access & & PDF & PDF \\
\hline MyMed Wall & Universal access & & $C C D, C-C D A$ & PDF, CCD, C-CDA \\
\hline My Doclopedia PHR & & & & PDF \\
\hline
\end{tabular}




\section{References}

1. Osborn R, Moulds D, Squires D, Doty MM, Anderson C. International survey of older adults finds shortcomings in access, coordination, and patient-centered care. Health Aff (Millwood) 2014; 33(12): 2247-2255. doi: 10.1377/hlthaff.2014.0947. PubMed PMID: 25410260.

2. Olsen RM, Hellzen O, Skotnes LH, Enmarker I. Breakdown in informational continuity of care during hospitalization of older home-living patients: a case study. Int J Integr Care 2014; 14: e012. PubMed PMID: 24868195; PubMed Central PMCID: PMC4027933.

3. Dykes PC, Samal L, Donahue M, Greenberg JO, Hurley AC, Hasan O, O’Malley TA, Venkatesh AK, Volk LA, Bates DW. A patient-centered longitudinal care plan: vision versus reality. J Am Med Inform Assoc 2014; 21(6): 1082-1090. doi: 10.1136/amiajnl-2013-002454. PubMed PMID: 24996874; PubMed Central PMCID: PMC4215040.

4. Bowles KH, Pham J, O'Connor M, Horowitz D. Information deficits in home care: a barrier to evidencebased disease management. HHCMP 2010; 22(4): 278-285.

5. Connecting for Health, Markle Foundation. The personal health working group: final report. New York, NY: Markle Foundation[Internet]. 2003 [cited 2015 December 1]. 58 p. Available from: http://www. markle.org/publications/1429-personal-health-working-group-final-report.

6. myPHR [Internet]. Chicago, IL: The American Health Information Management Association; c2015 [cited 2015 November 5]. Available from: http://www.myphr.com/.

7. What is a personal health record? [Internet]. Washington: Department of Health and Human Services; 2013 [cited 2015 November 30]. Available from: https://www.healthit.gov/providers-professionals/faqs/ what-personal-health-record.

8. Turvey C, Klein D, Fix G, Hogan TP, Woods S, Simon SR, Charlton, M, Vaughan-Sarrazin M, Zulman DM, Dindo L, Wakefield B, Graham G, Nazi K. Blue Button use by patients to access and share health record information using the Department of Veterans Affairs' online patient portal. J Am Med Inform Assoc 2014; 21(4): 657-663. doi: 10.1136/amiajnl-2014-002723. PubMed PMID: 24740865; PubMed Central PMCID: PMC4078285.

9. Dey JG, Johnson M, Pajerowski W, Tanamor M, Ward A. Home Health Study Report. Baltimore: Centers for Medicare and Medicaid Services, 2011.

10. Choose a PHR [Internet]. Chicago, IL: the American Health Information Management Association c2015 [cited 2015 November 5]. Available from: http://myphr.com/resources/choose.aspx.

11. Carrion Senor I, Fernandez-Aleman JL, Toval A. Are personal health records safe? A review of free webaccessible personal health record privacy policies. J Med Internet Res 2012; 14(4): e114. doi: 10.2196/jmir.1904. PubMed PMID: 22917868; PubMed Central PMCID: PMC3510685.

12. Pai HH, Lau F, Barnett J, Jones S. Meeting the health information needs of prostate cancer patients using personal health records. Curr Oncol 2013; 20(6): e561-e569. doi: 10.3747/co.20.1584. PubMed PMID: 24311957; PubMed Central PMCID: PMC3851353.

13. Millerick Y. Case study 6: an account of a patient's journey following a diagnosis of left ventricular systolic dysfunction of ischaemic aetiology. In: Stewart S, Blue L, editors. Improving Outcomes in Chronic Heart Failure: A practical guide to specialist 2nd ed. London: BMJ Publishing Group; 2004. p. 226-32.

14. Administration on Aging, Administration for Community Living. A Profile of Older Americans: 2012. Washington: U.S. Department of Human and Health Services; 2012. 14 p.

15. Hines AL, Barrett ML, Jiang HJ, Steiner CA. Conditions with the largest number of adult hospital readmissions by payer, 2011. Rockville (MD): The Agency for Healthcare Research and Quality, 2014.

16. Medem iHealthRecord [Internet]. Mountain View, CA: Intuit; c2015 [cited 2015 July 15]. Available from: http://www.medemphr.mymedfusion.com.

17. MyWellnessHub [Internet]. [cited 2015 July 15]. Available from: http://phr.emrystick.com.

18. MiVIA: Connecting Patients and Clinicians Nationwide. [Internet]. Sonoma, CA: MiVIA; c2013. [cited 2015 July 15]. Available from: https://www.mivia.org.

19. MyHealthFolders.com [Internet]. St. Louis, MO: BJC; c2015 [cited 2015 July 15]. Available from: https://www.myhealthfolders.com.

20.MyChart [Internet]. Verona, WI: Epic Systems; c1999-2013. [cited 2015 July 15]. Available from: https://www.mychartlink.com/MyChart/.

21.ZebraHealth [Internet]. Kirkland, WA: ZebraHealth Inc. [cited 2015 July 15]. Available from: https://www.zebrahealth.com.

22. About My Health: Experience. Learn. Share [Internet]. Nottingham, UK: EDIO Healthcare. [cited 2015 July 15]. Available from: http://www.aboutmyhealth.org/.

23. MedicAlert Foundation [Internet]. Turlock, CA: MedicAlert Foundation. [cited 2015 July 15]. Available from: http://www.medicalert.org/. 
24.dLife: It's your diabetes life [Internet]. LifeMed Media; c2015. [cited 2015 July 15]. Available from: http://www.dlife.com.

25.Zweena [Internet]. Hopewell, NJ: Zweena Health. [cited 2015 July 15]. Available from: http://zweenahealth.com/.

26. No More Clip Board [Internet]. Fort Wayne, IN: No More Clipboard; c2015. [cited 2015 November 5]. Available from: https://www.nomoreclipboard.com/.

27.Healthspek [Internet]. Healthspek; c2015. [cited 2015 November 5]. Available from: http://www.health spek.com/.

28. Health Companion [Internet]. San Diego, CA: Health Companion, Inc.; c 2009-2015. [cited 2015 November 5]. Available from: https://www.healthcompanion.com/.

29. HealthVault [Internet]. Redmond, WA: Microsoft; c2015. [cited 2015 November 5]. Available from: https://www.healthvault.com/us/en.

30. RememberItNow [Internet]. Orinda, CA: RememberItNow LLC. [cited 2015 November 5]. Available from: http://www.rememberitnow.com/index.php.

31.MyMedWall [Internet]. [cited 2015 November 5]. Available from: https://secure.mymedwall.com/phr/.

32. Doclopedia [Internet]. Phoenix, AZ: Doclopedia Marketplace Development. [cited 2015 November 5]. Available from: https://www.doclopedia.com/.

33. Wells S, Rozenblum R, Park A, Dunn M, Bates DW. Personal health records for patients with chronic disease: a major opportunity. Appl Clin Inform 2014; 5(2): 416-429. doi: 10.4338/ACI-2014-01-RA-0002. PubMed PMID: 25024758; PubMed Central PMCID: PMC4081745.

34. Studeny J, Coustasse A. Personal health records: is rapid adoption hindering interoperability? Perspect Health Inf Manag. 2014;11:1e. PubMed PMID: 25214822; PubMed Central PMCID: PMC4142513.

35. Smith A. Older Adults and technology use: adoption is increasing, but many seniors remain isolated from digital life. Washington, DC: Pew Research Center, 2014. 27 p.

36. Gu Y, Day K. Propensity of people with long-term conditions to use personal health records. Stud Health Technol Inform 2013; 188: 46-51. PubMed PMID: 23823287.

37.Prey J, Ryan B, Quian M, Restaino S, Bakken S, Feiner SK, Schnall R, Hripcsak G, Han J, Vawdrey D. Interim Results of a Randomized Controlled Trial on Inpatient Engagement. AMIA Annu Symp Proc 2015.

38. Sockolow PS, Bowles KH, Adelsberger MC, Chittams JL, Liao C. Impact of homecare electronic health record on timeliness of clinical documentation, reimbursement, and patient outcomes. Appl Clin Inform 2014; 5(2): 445-462. doi: 10.4338/ACI-2013-12-RA-0106. PubMed PMID: 25024760; PubMed Central PMCID: PMC4081747. 